\title{
Antiangiogenese in der Brustkrebstherapie
}

\author{
Gesprächsleitung: Andreas Schneeweiss ${ }^{a}$ \\ Teilnehmer: Joachim Bischoff ${ }^{b} \quad$ Bernd Gerber $^{c} \quad$ Nadia Harbeck $^{d}$ \\ aNationales Centrum für Tumorerkrankungen / Universitäts-Klinikum, Heidelberg, \\ bUniversitätklinikum Magdeburg, Universitätsfrauenklinik, Magdeburg, \\ 'Universitätsfrauenklinik und Poliklinik am Klinikum Südstadt der Hansestadt Rostock, \\ ${ }^{\mathrm{d} B r u s t z e n t r u m ~ d e r ~ U n i v e r s i t a ̈ t ~ M u ̈ n c h e n, ~ K l i n i k u m ~ G r o s s h a d e r n ~ d e r ~ L M U ~ M u ̈ n c h e n, ~ G e r m a n y ~}$
}

Frage 1: Welchen Stellenwert hat die antiangiogene Therapie beim Mammakarzinom derzeit im klinischen Alltag?

Bischoff: Bevacizumab-basierte Regime stellen eine Bereicherung der Optionen beim metastasierten HER2-negativen Mammakarzinom dar. Sie bieten vor allem dann eine nebenwirkungsarme Alternative, wenn bei einer Hochrisiko-Situation eine Polychemotherapie indiziert ist. Aus sozioökonomischen Gründen sollte die Indikation allerdings kritisch gestellt werden, da prädiktive Faktoren für den Einsatz des kostenintensiven Antikörpers bislang fehlen. Als limitierender Faktor kann außerdem gelten, dass die Zulassung auf die Erstlinien-Therapie in Kombination mit Paclitaxel oder neuerdings Capecitabin beschränkt ist.

Gerber: Bei HER2-normalen metastasierten Patientinnen mit Indikation zu einer Chemotherapie stellt die Kombination von Bevacizumab und Chemotherapie - je nach Vortherapie mit Paclitaxel oder Capecitabin - den Therapiestandard dar. Das Gesamtüberleben wird zwar nicht verbessert, aber die klinischen Responseraten sind unter der Kombination am höchsten. Dieses rasche und deutliche Ansprechen ist in der Therapie metastasenbedingter Beschwerden von immenser Bedeutung. Denn «Ziel in der Behandlung des metastasierten Mammakarzinoms ist die Wiederherstellung und Erhaltung der Lebensqualität durch Linderung von metastasenbedingten Beschwerden, die Erhaltung des sozialen Umfeldes und nicht die Verlängerung des Gesamtüberlebens!» [Gerber B, Freund M, Reimer T: Recurrent breast cancer: treatment strategies for maintaining and prolonging good quality of life. Dtsch Ärztebl Int 2010;107:85-91].
Harbeck: Von allen bisher in klinischen Studien untersuchten Antiangiogenese-Substanzen hat es allein der monoklonale Antikörper Bevacizumab zur Zulassung geschafft. Bevacizumab stellt gemeinsam mit den Chemotherapie-Partnern Paclitaxel oder Capecitabin eine zugelassene, leitliniengerechte und evidenzbasierte Therapieoption für die ErstlinienSituation beim metastasierten Mammakarzinom dar. Die Kombination führt zu einer Verlängerung des progressionsfreien Überlebens, jedoch in den drei Zulassungsstudien nicht zu einer Verlängerung des Gesamtüberlebens. Ein eindeutig favorisiertes Patientenkollektiv gibt es nicht. Während in der neoadjuvanten Therapie vielversprechende Daten zum pathologisch kompletten Ansprechen (pCR) beim tripel-negativen Mammakarzinom vorliegen, so zeigen die Daten beim metastasierten Mammakarzinom eine Wirkung unabhängig vom Hormonrezeptorstatus.

\section{Frage 2: Was können wir aus den Rückschlägen bei der klinischen Entwicklung der antiangiogenen Therapie lernen?}

Bischoff: Derartige Rückschläge waren vor allem in zweierlei Hinsicht zu verzeichnen:

a) Manche Vertreter der neuen Substanzklasse der Multikinasehemmer (z.B. Sunitinib, Mosetanib) wurden frühzeitig Bestandteil multinationaler Phase-III-Studien, ohne dass im Vorfeld die biologischen Grundlagen beim Mammakarzinom hinreichend überprüft wurden. Zudem lagen Daten aus den erforderlichen Vorläuferstudien nur im begrenzten Ausmaß vor. Nicht zuletzt auch wegen der überlappenden Toxizitäten mit den meisten gängigen Zytostatika sollte die weitere Evaluation von Multikinasehemmern beim Mammakarzinom zurückhaltend erfolgen.

\section{KARGER \\ Fax +497614520714 \\ Information@Karger.de}

www.karger.com (c) 2011 S. Karger GmbH, Freiburg

$1661-3791 / 11 / 0066-0485 \$ 38.00 / 0$

Accessible online at:

www.karger.com/brc
Prof. Dr. med. Andreas Schneeweiss

Nationales Centrum für Tumorerkrankungen

Universitäts-Klinikum

Im Neuenheimer Feld 460, 69120 Heidelberg, Germany

Tel. +49 6221 56-36051, Fax -7920

andreas.schneeweiss@med.uni-heidelberg.de 
b) Bei dem Versuch, die Bevacizumab-Therapie zu optimieren, wurde bislang in erster Linie auf den Synergismus der Substanz mit verschiedensten Zytostatika gesetzt. Die entsprechenden Ergebnisse legen jedoch den Schluss nahe, dass auf diese Weise zumindest im metastasierten Setting wohl keine weitere Verbesserung der Effizienz antiangiogener Therapien erzielt werden kann.

Gerber: Obwohl Metastasenwachstum mit Vaskularisation einhergehen muss, bedeutet dies nicht, dass alle Tumortypen gleichmäßig auf eine antiangiogenetische Therapie ansprechen. Eine Substanz für alle Mammakarzinome wird es nicht mehr geben. Insofern müssen zukünftige Studien nach der Tumorbiologie stratifizieren. Für alle neuen antiangiogenetischen Substanzen wäre die Identifikation eines Targets oder bestimmter Genotypen (z.B. VEGF-Allele, Angiopoietine, VEGF-Rezeptorsysteme) wünschenswert.

Harbeck: Eine Entwicklung einer zielgerichteten Therapie ohne einen dazugehörigen Biomarker, d.h. klinisch anwendbaren Nachweis der Zielstruktur, ist wenig sinnvoll. Mehrere Phase-III-Studien an unselektierten Patientenkollektiven mussten wegen fehlender Effektivität geschlossen werden eine solche Verschwendung von Patienten-Engagement und Ressourcen muss der Vergangenheit angehören. In Zukunft benötigen wir intelligente Studiendesigns, die potentielle Biomarker von Anfang an mit einbeziehen.

\section{Frage 3: Wie sollte die antiangiogene Therapie beim Mammakarzinom weiter entwickelt werden?}

Bischoff: Angesichts des komplexen Netzwerkes bereits bekannter angiogener Faktoren liegt es nahe, die Anti-VEGFTherapie mit anderen Substanzen zu kombinieren, die angiogene Signalwege blockieren (z.B. mTOR-Inhibitoren oder metronomische Chemotherapie). Die Aktivierung proangiogener Faktoren scheint als Feedback-Schleife bei der Bevacizumab-Resistenz eine wichtige Rolle zu spielen. Andererseits lassen die Daten beim tripel-negativen Mammakarzinom die Hoffnung zu, dass Bevacizumab insbesondere bei dieser Subgruppe einen Stellenwert besitzt und in bewährter Kombination mit etablierten Zytostatika weiter prospektiv untersucht werden sollte. Gleichzeitig ist von großer klinischer Relevanz und bisher noch ungeklärt, ob der VEGF-Antikörper im Falle einer Progression in Analogie zum TBP-Konzept bei Trastuzumab weiter gegeben werden sollte. Generell ist zu überlegen, ob die klinische Erprobung neuer Angiogenese-Hemmer nicht bereits frühzeitig auf neoadjuvante Studien zurückgreift, um dadurch schneller als bisher aussagekräftige Informationen zur biologischen Aktivität der jeweiligen Substanz zu erhalten, bevor eine große Zahl von Patienten in ein Phase-III-Programm bei metastasierter Erkrankung eingeschleust wird.
Gerber: Antiangiogene Substanzen werden aus den bei Frage 2 genannten Gründen auch in Zukunft eine wesentliche Säule in der Therapie des Mammakarzinoms darstellen. Neben der Hemmung der Gefäßneusprossung sollte die Gefäßausreifung bis hin zur Rückbildung der unreifen Tumorgefäße durch antiangiogenetische Substanzen untersucht werden. Dazu sollten Substanzen entwickelt werden, die verschiedene Angiopoietine aber auch andere Wachstumsfaktoren, wie z.B. PDGF (platelet derived growth factor) modulieren.

Harbeck: Wie bereits gesagt, benötigen wir dringend Tumoroder Blut-basierte Biomarker, die das Ansprechen auf eine antiangiogene Therapie vorhersagen können. Ein möglicher wegweisender Ansatz ist die geplante Studie, bei der die Wirkung von Bevacizumab an Patientengruppen, die anhand des Plasma-VEGF-A-Spiegels unterteilt wurden, untersucht wird. Eine weitere vielversprechende Möglichkeit ist die Untersuchung des frühen Ansprechens mittels molekularer Bildgebung (konventionelles FDG-PET), aber auch neue, Integrin-basierte Tracer (RGD) können hier bereits nach einem Therapiezyklus Hinweise auf ein späteres Ansprechen geben - in der GALADON-Studie der WSG werden wir im nächsten Jahr die klinische Wertigkeit der PET-Untersuchung bei neoadjuvanter Therapie mit Docetaxel und Bevacizumab untersuchen.

\section{Frage 4: Welches sind die vielversprechendsten Angriffspunkte für eine antiangiogene Therapie?}

Bischoff: Noch ist der Wachstumsfaktor VEGF das am besten untersuchte Target im Bereich der Angiogenese-Forschung. Erste klinische Erfahrungen liegen außerdem inzwischen vor allem für weitere angiogenesespezifische Wachstumsfaktorrezeptoren wie PDGF, FGF und PIGF vor, allerdings ohne bisherige Hinweise darauf, dass deren Blockade beim Mammakarzinom eine Relevanz besitzt. Diese Daten beziehen sich jedoch in erster Linie auf den Einsatz von Multi-TargetInhibitoren wie Sunitinib und Motesanib. Noch befinden sich einzelne Präparate aus dieser Substanzklasse, wie etwa Sorafenib, in der fortgeschrittenen Phase der klinischen Evaluation (metastasiertes Setting). Ansonsten ist derzeit nicht klar erkennbar, welche neuen molekularen Targets besonders aussichtsreiche Kandidaten für eine antiangiogenetische Therapie darstellen. Manches spricht dafür, dass die Blockade der Angiopoetin-Tie-Rezeptor-Achse als erfolgversprechender Ansatz anzusehen ist.

Gerber: VEGF steht in der Angiogenesekaskade sehr weit oben. Insofern gibt es zahlreiche weitere Targets in der darunter ablaufenden Kaskade. Tyrosinkinaseinhibitoren sind weniger spezifisch und hemmen neben den VEGFRezeptoren zusätzlich auch andere, mit ihnen verwandte Tyrosinkinasen. Eine Vielzahl dieser neuen Substanzen sind 
in der klinischen Testung, allerdings sollten zunächst die Ergebnisse abgewartet werden.

Harbeck: Wir wissen wenig darüber, ob der Ligand VEGF wirklich der beste Ansatzpunkt für eine antiangiogene Substanz ist, oder ob vielmehr die Rezeptoren hier die besseren Zielstrukturen sind. Multityrosinkinaseinhibitoren haben ein breiteres Hemmspektrum - bisher fehlt hier jedoch der klinische Beweis, dass dieses breitere Angriffsspektrum sich in einer klinisch überlegenen Therapie manifestiert.

\section{Frage 5: Welche konkreten Fragen haben die Kliniker an die Grundlagenforscher im Zusammenhang mit der Weiterentwicklung der antiangiogenen Therapie?}

Bischoff: Die Komplexität des angiogenen Netzwerkes legt nahe, dass das VEGF-Targeting in der jetzigen Form nur den Beginn der Ära der antiangiogenetischen Therapien markieren kann. Einerseits bedürfen die Bevazizumab-assoziierten Resistenzmechanismen der weiteren Aufklärung. Ungeachtet der bisherigen Rückschläge muss außerdem weiter nach prädiktiven Faktoren gesucht werden. Außerdem sollte verstärkt nach funktionell bedeutsamem Cross-Talks zwischen dem VEGFR-Signaling und anderen Angiogensebezogenen Signalwegen Ausschau gehalten werden. Wie bereits erwähnt, scheint beispielsweise ein Synergismus zwischen der mTOR-Inhibition und VEGF-Targeting zu bestehen. Positive Phase-II-Daten liegen diesbezüglich beim Nierenzellkarzinom vor.

Gerber:

- Ist es wirklich sinnvoll, alle Tumorgefäße zu zerstören, um den Tumor «trockenzulegen»? Kann durch die starke Hypoxie im Tumor nicht auch die Resistenzentwicklung und der Prozess der Metastasierung beschleunigt werden?

- Wie wirkt die Kombination von zwei oder mehreren unterschiedlich angreifenden antiangiogenetischen Substanzen oder die Kombination mit Phototherapie?

- Welche Bedeutung haben antiangiogenetische Substanzen in der Verhinderung einer Metastasierung?
Harbeck: Neben der optimalen Substanz haben wir viele Fragen zum besten Einsatz einer antiangiogenen Therapie beim Mammakarzinom. In der metastasierten Situation ist die optimale Therapiedauer unklar - bisher wird bis zum Progress therapiert, aber dann die Chemotherapie umgestellt, ohne Bevacizumab weiterzugeben - fraglich ist, ob hier ein Treatment-beyond-progression-Vorgehen sinnvoll ist. Ein klinisch relevanter Rebound-Effekt konnte in den bisherigen Studien nicht nachgewiesen werden. Auch hier benötigen wir weiter Daten, v.a. auch mit Tyrosinkinaseinhibitoren, um diese Frage zu klären. Je weiter sich die Metastasen entdifferenzieren, umso größer wird der Einfluss vielfältiger Strukturen auf die Tumorprogression - eine frühzeitige Kombination mit einem anderen Biologicals (z.B. mTOR-Inhibitor) wäre hier zumindest sinnvoll. In der adjuvanten Situation ist bisher unklar, ob Bevacizumab eine adjuvante Wirkung hat und ob diese Wirkung sich über den Behandlungszeitraum hinaus erstreckt. Die noch ausstehenden Daten aus den großen internationalen Studien (BEATRICE, BETH) werden dringend erwartet.

\section{Teilnehmer}

Dr. med. Joachim Bischoff

Universitätklinikum Magdeburg A.ö.R.

Universitätsfrauenklinik

Gerhart-Hauptmann-Straße 35

39108 Magdeburg, Germany

Joachim.Bischoff@med.ovgu.de

Prof. Dr. med. Bernd Gerber

Universitätsfrauenklinik und Poliklinik

am Klinikum Südstadt der Hansestadt Rostock

Südring 81, 18059 Rostock, Germany

Tel. +49 381 4401-4500, Fax -4599

bernd.gerber@med.uni-rostock.de

Prof. Dr. med. Nadia Harbeck

Brustzentrum der Universität München

Klinikum Grosshadern der LMU München Marchioninistr. 15, 81377 München, Germany

Tel. +49 89 7095-4531, Fax -8892

nadia.harbeck@med.uni-muenchen.de 